\title{
Relación entre PIB Municipal y Características de Accesibilidad Territorial hacia Fincas Productoras de Cinco Frutales: Tomate de Árbol, Guayaba, Mora, Maracuyá y Lulo
}

\author{
Diego A. Escobar, Jhonny A. Tamayo y Camilo Younes \\ Facultad de Ingeniería y Arquitectura, Universidad Nacional de Colombia, Sede Manizales, \\ Carrera 27 \# 64 - 60, Campus Palogrande, Bloque Posgrados, Oficina I - 408, Colombia \\ (e-mail: daescobarga@unal.edu.co, jatamayoar@unal.edu.co, cyounesv@unal.edu.co)
}

Recibido Ago. 25, 2017; Aceptado Nov. 7, 2017; Versión final Dic. 1, 2017, Publicado Abr. 2018

\begin{abstract}
Resumen
En este estudio se aplican técnicas geoestadísticas de interpolación espacial con el fin de evaluar la posición geográfica de fincas productoras de cinco tipos de frutales comerciales andinos: Mora, Maracuyá, Tomate de árbol, Lulo y Guayaba. Se ha desarrollado un proceso investigativo tendiente a identificar posibles potencialidades y desventajas que ofrece el sistema de infraestructura del trasporte disponible en la zona de estudio, evaluando los tiempos medios de viaje hacia zonas de producción y su cobertura en relación a variables socioeconómicas como el Producto Interno Bruto (PIB) propio de cada municipio. Este tipo de análisis permite identificar zonas con deficiencias en materia de infraestructura del transporte con el objeto de priorizar inversiones.
\end{abstract}

Palabras clave: accesibilidad; cobertura; frutales andinos; geoestadística; tiempo medio de viaje; transporte

\section{Relationship between Municipal GDP and Territorial Accessibility Characteristics to Farms, Producers of Five Fruits: Tomato Tree, Guava, Blackberry, Passion Fruit, and Lulo}

\begin{abstract}
Geostatistical techniques of spatial interpolation are applied in this study in order to evaluate the geographical location of farms that produce five types of andean commercial fruits: Blackberry, Passion fruit, Tomato Tree, Lulo and Guava. An research process has been developed to identify possible potentialities and disadvantages offered by the transport infrastructure system available in the study area, evaluating the average travel times to production zones and their coverage with respect to socioeconomic variables as the Gross Domestic Product (GDP) of each municipality. This type of analysis makes it possible to identify areas with deficiencies in transport infrastructure with the target to prioritize investment.
\end{abstract}




\section{INTRODUCCIÓN}

El presente estudio es un componente del proyecto de investigación aplicada titulado: "Modelo de plataforma de aprovechamiento integral, adición de valor y competitividad de frutales comerciales andinos", el cual ha sido desarrollado por la Universidad Nacional de Colombia sede Manizales y financiado por el Banco Interamericano de Desarrollo (BID). El objetivo principal del estudio consiste en determinar qué potencialidades o desventajas refiere la ubicación geográfica de las fincas productoras de cinco tipos de frutales andinos (Mora, Maracuyá, Tomate de árbol, Lulo y Guayaba) en relación con las condiciones de accesibilidad territorial que brinda la infraestructura vial existente, evaluando la cobertura de variables socioeconómicas como el Producto Interno Bruto (PIB) propio de cada municipio. La zona de estudio hace parte de la región centro occidente de Colombia (Ver Figura 1), específicamente abarca el Departamento de Caldas y algunos municipios del Departamento de Risaralda (Pereira, Santa Rosa de Cabal, Dosquebradas, La Virginia, Quinchía, Guática y Marsella); área para la cual se debió caracterizar la red de infraestructuras del transporte existente, que conecta con las 31 fincas productoras que son objeto de análisis.

La infraestructura vial y del transporte se configura como uno de los principales soportes de dinámica y desarrollo para una comunidad; es así que gracias a las conexiones viales se hace posible la comercialización y distribución de materias primas, bienes y servicios desde las zonas urbanas hacia las zonas rurales y viceversa. Las características físicas y operacionales de las vías constituyen factores altamente influyentes y de utilidad para orientar esfuerzos en materia de inversión y localización geográfica de nueva infraestructura y equipamientos, es por eso que los planificadores urbanos enfatizan de manera constante el efecto que tiene la accesibilidad sobre el desarrollo del suelo (Hansen, 1959). Las mejoras en las redes de transporte permiten el crecimiento de la economía, reducen costos de producción e incrementan la competitividad de todos los sectores de la economía (Gutiérrez, 2001).

La definición más concreta y clásica del concepto "accesibilidad" es la establecida en la sexta década del siglo pasado por Hansen (1959), como: "...the potential of Opportunities for interaction."; de forma general, el concepto se entiende como una medida que indica la dificultad o facilidad que tiene las comunidades 0 actividades para conectarse entre un origen y un destino (Morris et al., 1978), teniendo que dicha comunicación se materializa a través del uso de uno o múltiples medios de transporte. Ahora bien, desde el punto de vista de análisis de redes, un estudio de accesibilidad geográfica consiste básicamente en calcular el tiempo de viaje que debe invertirse para llevar a cabo un desplazamiento entre puntos de interés haciendo uso de la infraestructura de transporte disponible. El concepto de accesibilidad, ampliamente utilizado en diversos campos de las ciencias de la tierra como el planeamiento urbano y regional, se ha difundido desde primeras décadas del siglo pasado en aspectos relacionados con estudios de localización y desarrollo económico (Rietveld y Nijkamp, 1993; Mackinnion et al., 2008; Batty, 2009), análisis demográfico (Kotavaara, 2011), análisis de las facilidades ofrecidas por la infraestructura de transporte (Mitchell y Rapkin, 1954; Martínez et al, 2017), estudios relacionados con la cohesión social (López et al., 2008), localización de servicios bibliotecarios (Park, 2012), análisis de sectores educativos (Younes et al., 2016), geomárketing (Zuluaga y Escobar, 2017), sostenibilidad (Vega, 2011; Escobar et al., 2015a), operatividad de modos de transporte (Escobar et al., 2012; Montoya et al., 2017), entre otros campos.

Las medidas de accesibilidad son de fácil interpretación para funcionarios públicos e investigadores y representan un criterio útil para guiar decisiones en políticas de uso del suelo, ordenamiento territorial y políticas de infraestructura (Geurs, K. y Van Wee, B., 2004). En este sentido, para el sector de la agricultura resulta bastante útil evaluar las condiciones de accesibilidad que ofrece la infraestructura del transporte, con el objetivo de optimizar el proceso productivo, específicamente el eslabón correspondiente al traslado de los productos agrícolas, lo cual redundaría en la reducción de costos. A su vez, vale la pena resaltar que la rugosidad propia de la superficie de rodadura de las vías produce un efecto oscilatorio sobre los vehículos con niveles de aceleración del orden de 0,25 a 0,5 G (Van Zeebroeck et al., 2007) representando una causa potencial de deterioro para los productos. En consecuencia es natural inferir que entre más prolongado sea el periodo de transporte, existirá un tiempo de exposición mayor y en consecuencia una afectación considerable para la calidad del producto. Cabe precisar que el tiempo de viaje es sólo una de las causas que influye en la disminución de la calidad, influyen también el tipo de vehículo, daños por impacto en el proceso de empaque, la susceptibilidad al daño propia de cada fruta, entre otros (Van Zeebroeck et al., 2006). Considerando que los resultados derivados del cálculo de accesibilidad territorial representan a su vez un impacto social y económico (Gutiérrez et al., 2010); en el desarrollo del presente artículo se establece una relación entre los tiempos medios de viaje calculados y los valores de PIB (Producto interno bruto) propios de cada municipio de la zona de estudio (Ver Figura 2), el cual representa un indicador directo de su grado de desarrollo, con el objetivo de evaluar la cobertura que ofrece la red vial con respecto a este importante indicador económico. 

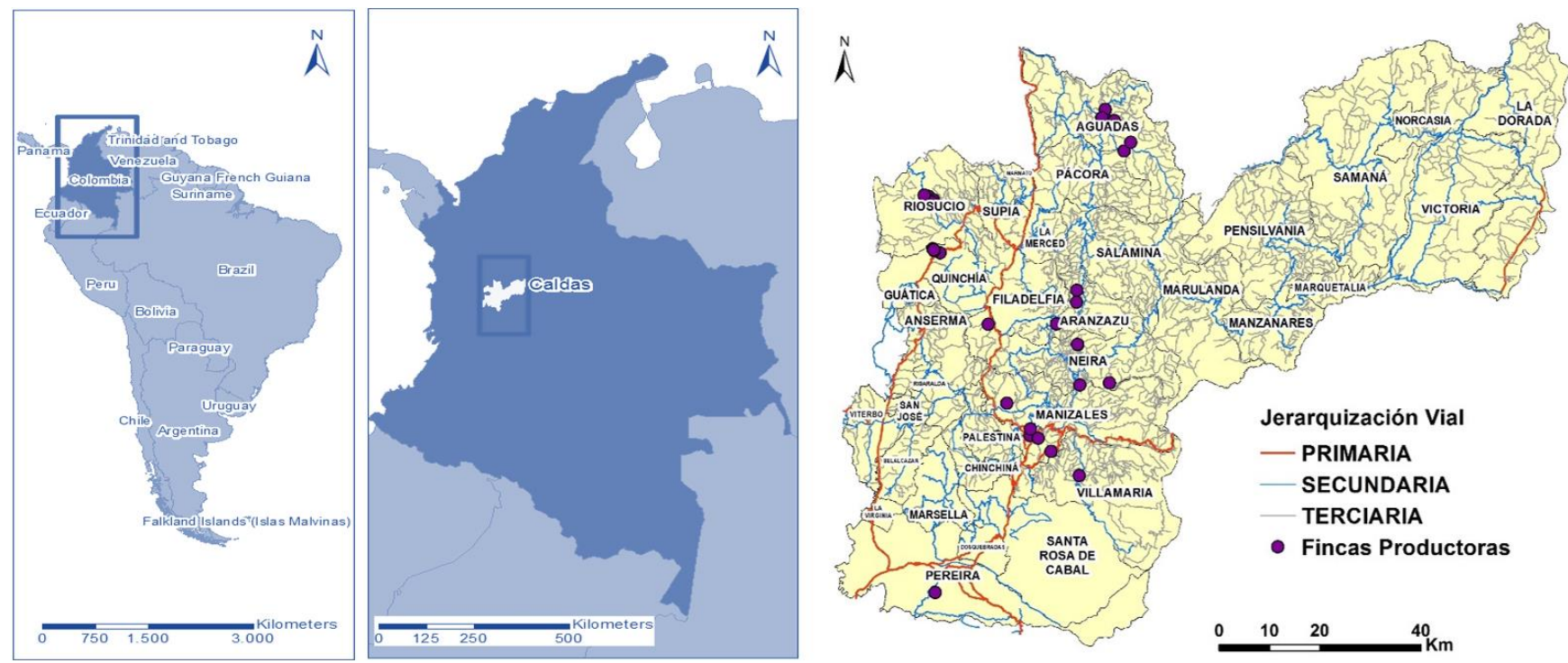

Fig. 1. Localización geoespacial de la zona de estudio.

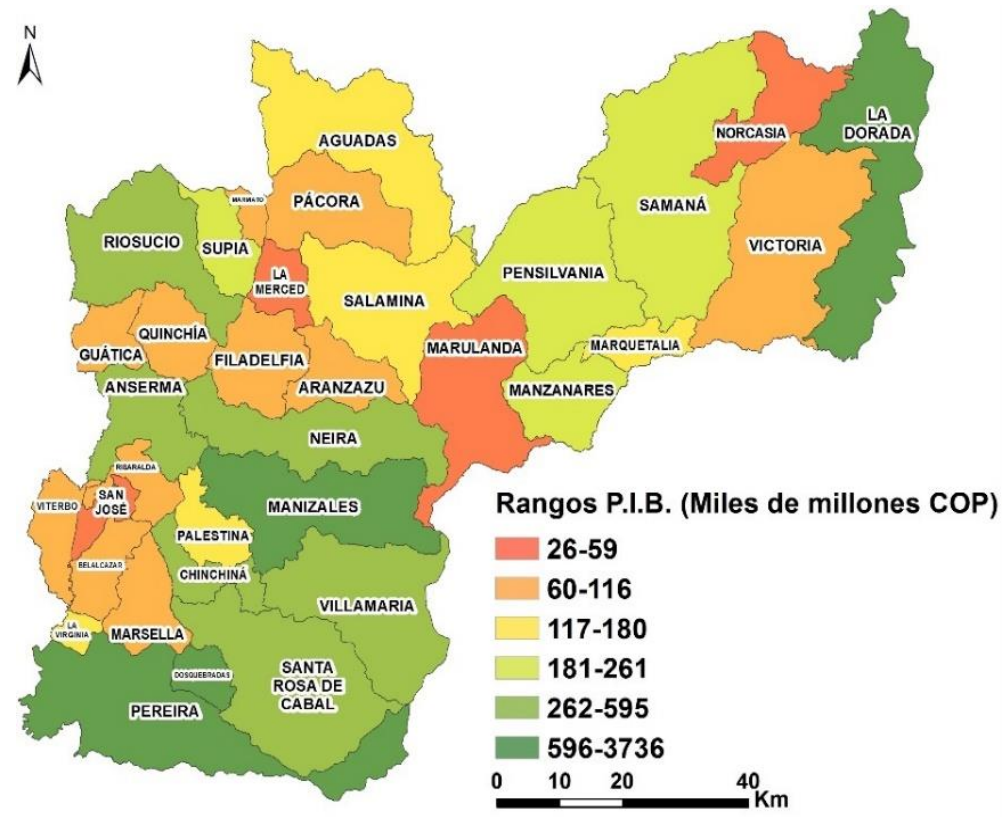

Fig. 2. Rangos de Producto Interno Bruto en la zona de estudio

De igual manera, con el fin de determinar el impacto social, se considera información demográfica, específicamente el número de habitantes y se calcula en qué medida la red vial al interior del área de análisis representa un beneficio para los pobladores, entendiendo que el comportamiento y el desarrollo de la actividad humana se encuentra relacionado con sus necesidades de viajar (Fox, 1995). Los resultados arrojados por el estudio constituyen una herramienta de planificación que permite identificar zonas con condiciones desfavorables de accesibilidad y en base a ello, plantear sugerencias de priorización en la inversión pública, orientadas al mejoramiento de la malla vial, generando un impacto positivo sobre todos los sectores de la economía regional.

\section{METODOLOGÍA}

La presente investigación se ha desarrollado en cuatro etapas (Ver Figura 3): i) digitalización y caracterización de la infraestructura del transporte; ii) localización geoespacial de las fincas productoras; iii) cálculos y análisis de accesibilidad geográfica; iv) cálculo y análisis de cobertura.

Etapa 1. Digitalización y caracterización de la infraestructura del transporte: Esta etapa constituye el pilar principal para el desarrollo del estudio y consiste en la digitalización en el sistema de información geográfica SIG (ArcMap en este caso) de cada uno de los segmentos viales en la zona de estudio asignando los respectivos atributos de longitud, velocidad de operación, direccionalidad, jerarquización y tiempo medio de 
viaje (Ver Figura 1). Cabe precisar que la información proviene de diferentes fuentes, como el Departamento Administrativo Nacional de Estadística (DANE), el Instituto Nacional de Vías (INVIAS), así como información primaria obtenida mediante la realización de viajes con equipos GPS a bordo, permitiendo el cálculo de las velocidades de operación en cada tramo de la red vial. La zona de estudio cuenta con una red vial de 8.162 $\mathrm{Km}$, de los cuales $446 \mathrm{Km}$ (5,5\%) corresponden a vías primarias, como la "Autopista del Café" que conecta a Manizales con los municipios de Chinchiná, Santa Rosa de Cabal, Dosquebradas y Pereira y Hacia el norte la vía que conecta a Manizales con el departamento de Antioquia. En cuanto a las vías secundarias, estas suman $1.879,9 \mathrm{Km}$ (23\%), la principal función de este tipo de vías es la de comunicar las cabeceras municipales. Por último, se encuentran las vías terciarias, las cuales totalizan 5.836,1 Km (71,5\%).

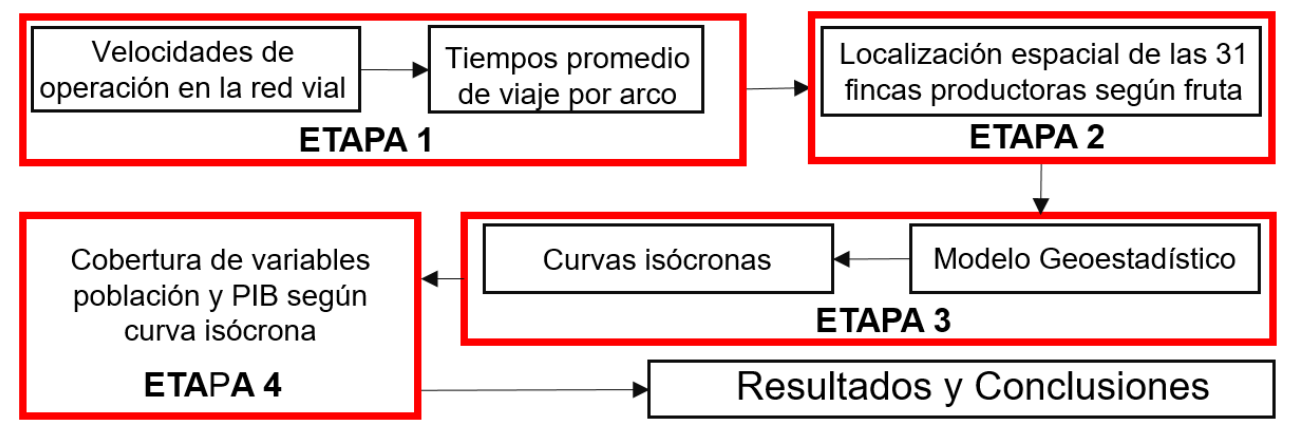

Fig. 3. Metodología aplicada

Etapa 2. Localización Geoespacial de las fincas productoras: Por medio de la información publicada en la página web "geográfica de las fincas consideradas en el presente estudio y las frutas producidas en cada una de ellas, (Mora, Maracuyá, Tomate de árbol, Lulo y Guayaba). www.frutalesandinos.com/" se ha realizado la consulta de los agricultores vinculados al proyecto, así como la localización de las fincas. En la Figura 1 se puede apreciar la localización de las fincas productoras consideradas en este estudio, se evidencia la concentración de éstas en la cercanía de los principales corredores viales; así mismo, es notoria la inexistencia de este tipo de predios productivos en el oriente caldense.

Etapa 3. Cálculos y análisis de accesibilidad geográfica: Una vez surtidas las dos primeras etapas, se procede al desarrollo de los cálculos de accesibilidad empleando el software ArcGis y su paquete de herramientas denominado "Network Analyst" y por medio del comando "Closest Facility", el cual emplea el algoritmo de Dijkstra para calcular el tiempo de viaje hacia la finca más cercana buscando optimizar la variable "tiempo de viaje" desde cada nodo de la red vial. El resultado de este procedimiento de cálculo es un vector de " $n$ " elementos, siendo " $n$ " el número de nodos que posee la red vial (11.188), este es el vector de tiempo medio de viaje. Posteriormente es necesario espacializar esta información de tipo puntual, para ello existen diversos métodos de interpolación de tipo geoestadístico, en este caso se ha empleado el método de Kriging el cual ajusta una función o modelo matemático para reflejar la correlación espacial entre los puntos de entrada para así explicar la variación superficial (Hengl et al, 2007). Las predicciones generadas por el modelo se validan estadísticamente mediante un semivariograma (Pardo-Igúzquita, 2007) y un modelo de covarianza (Goovaerts, 1999). La ecuación general del método se observa en la Expresión (1). Donde $Z_{0}$ se refiere al valor a pronosticar, $Z_{x}$ son los valores de los puntos conocidos, $W_{x}$ es el peso de los puntos conocidos respecto a los otros puntos del área geográfica a extrapolar según la variable tiempo medio de viaje y $S$ son los números de puntos conocidos. Para el cálculo de la semivarianza se aplica la Expresión (2), en la cual h es la distancia entre puntos, $n$ son números pares de puntos que se definen según la distancia entre ellos y $z$ son los valores de puntos conocidos (Phatarapon et al., 2015).

$$
\begin{aligned}
& Z_{0}=\sum_{i=1}^{S} Z_{x} w_{x} \\
& f(h)=\frac{1}{2 n} \sum_{i=1}^{n}\left[z\left(x_{i}\right)-z\left(x_{i}+h\right)\right]^{2}
\end{aligned}
$$

Etapa 4. Cálculo y análisis de cobertura: Una vez elaboradas las curvas isócronas, se procede a evaluar los niveles de cobertura que la infraestructura del transporte proporciona en cuanto al acceso a las fincas productoras y a su vez, se relaciona la distribución de los tiempos de viaje calculados con el número de habitantes y el PIB propio de cada municipio. Cabe precisar que la zona de estudio ocupa un área de 9.089 $\mathrm{Km}^{2}$, se compone de 34 municipios, que albergan un total de 1.833 .118 habitantes, las capitales de departamento, Manizales y Pereira, reúnen el 47,2\% de la población de la zona de análisis, con 21,6\% y $25,6 \%$ respectivamente; estos valores indican la alta concentración urbana que existe en esta zona de Colombia. 


\section{RESULTADOS Y DISCUSIÓN}

El resultado de los procedimientos de tipo geoestadístico son las curvas isócronas, las cuales ilustran el tiempo medio de viaje que debe ser invertido desde cualquier punto del área de estudio hacia la finca más cercana según la fruta que produce; gracias a este insumo fue posible identificar aquellas áreas que perciben mayores o menores beneficios en materia de accesibilidad territorial según la fruta que se produce.

\section{Fincas productoras de tomate de árbol}

En la Figura 4 se observan las curvas isócronas calculadas para evaluar la accesibilidad hacia las fincas productoras de tomate de árbol; cabe precisar que se cuenta con dos fincas de este tipo localizadas en la zona norte, concretamente en los municipios de Anserma y Riosucio. Se observa que las curvas van desde las 0,5 horas hasta las 6 horas. La zona noroccidente caldense refiere las condiciones más favorables, identificándose la influencia directa del corredor vial principal en el sentido norte sur y viceversa sobre la ampliación de las curvas hacia el Departamento de Risaralda, encontrando unos tiempos medios de viaje de hasta 3,5 horas desde municipios como Santa Rosa de Cabal y Pereira. Por otra parte, en el municipio de La Dorada (oriente) se encuentra cubierto por curvas de 5,5 horas y 6,0 horas indicándole como la zona con peores condiciones de accesibilidad en relación con la ubicación de este tipo de finca productora.

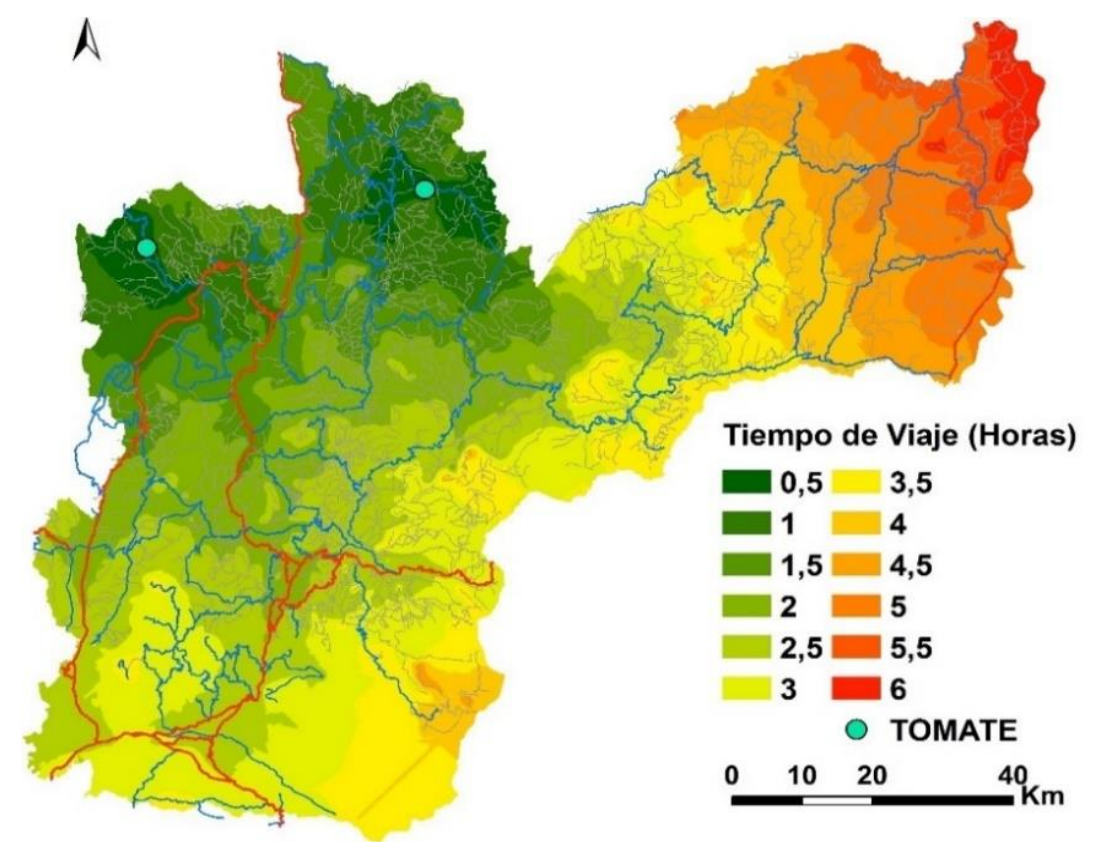

Fig.4. Curvas isócronas hacia las fincas productoras de Tomate de árbol

Analizando la cobertura de las variables PIB, área y población según curva isócrona (Ver Figura 5a) puede concluirse que el $50 \%$ de las tres variables se cubre invirtiendo un tiempo medio de viaje menor a 2,5 horas; de la misma forma, se observa que las tres variables presentan coberturas superiores al $80 \%$ con una inversión de tiempo medio de viaje de hasta 4 horas. Ahora bien, al disgregar los resultados de cobertura relacionados con el PIB, se observa que los municipio con PIB rango 3, es decir, con un rango medio de producción, son los que mejores condiciones de accesibilidad refieren en relación con el tiempo medio de viaje, al encontrarse más cerca del origen (Ver Figura 5b); por otra parte, se tiene que los municipios con PIB4 y PIB6, es decir, con una mayor producción, refieren condiciones de cobertura más desfavorables. Lo anterior indica que las fincas productoras de tomate de árbol se encuentran alejadas de los grandes centros de comercialización, entendidos éstos como los municipios de mayor PIB (Manizales, Pereira, Dosquebradas y La Dorada), lo cual se refuerza en que para bajos tiempos medios de viaje, es mayor la cobertura de la variable área que la cobertura de las variables Población y PIB, concluyendo bajas densidades poblacionales, mientras que para altos tiempos medios de viajes se identificarían altas densidades de población, lo cual se relaciona con los grandes centros de comercialización. 

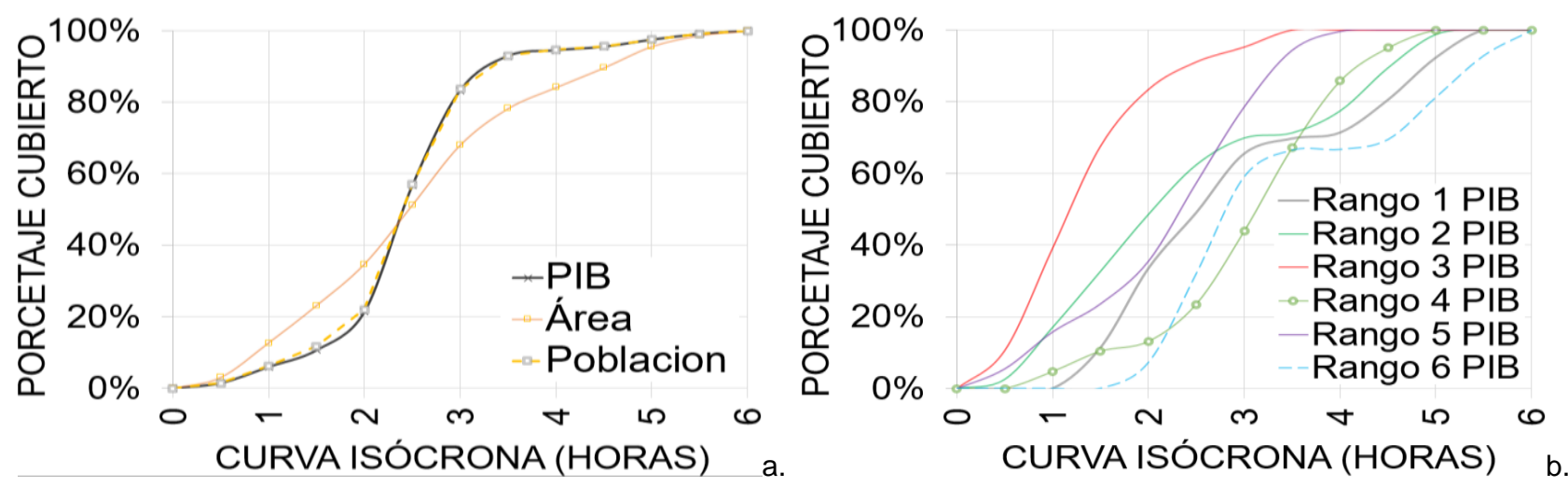

Fig. 5. a. Ojivas de cobertura de fincas productoras de Tomate de Árbol según PIB, área y población; b. Ojivas de cobertura según rangos de PIB.

\section{Fincas productoras de guayaba}

En la Figura 6 se observan las curvas isócronas obtenidas al evaluar la accesibilidad ofrecida por la red de infraestructuras del transporte hacia las fincas productoras de guayaba; se contó con la localización de cuatro fincas que producen este tipo de fruta, una ubicada al sur de la zona de estudio (Pereira), mientras las otras tres ubicadas un poco más al centro (Manizales y Palestina), muy cercanas todas a los principales ejes de movilidad. Toda el área de estudio se cubre con tiempos medios de viaje de hasta 7 horas; dada la localización de las fincas productoras de mora, se observa que es la zona del occidente caldense la que refiere las condiciones más favorables presentando tiempos medios de viaje de hasta 3 horas en el sector norte (Aguadas).

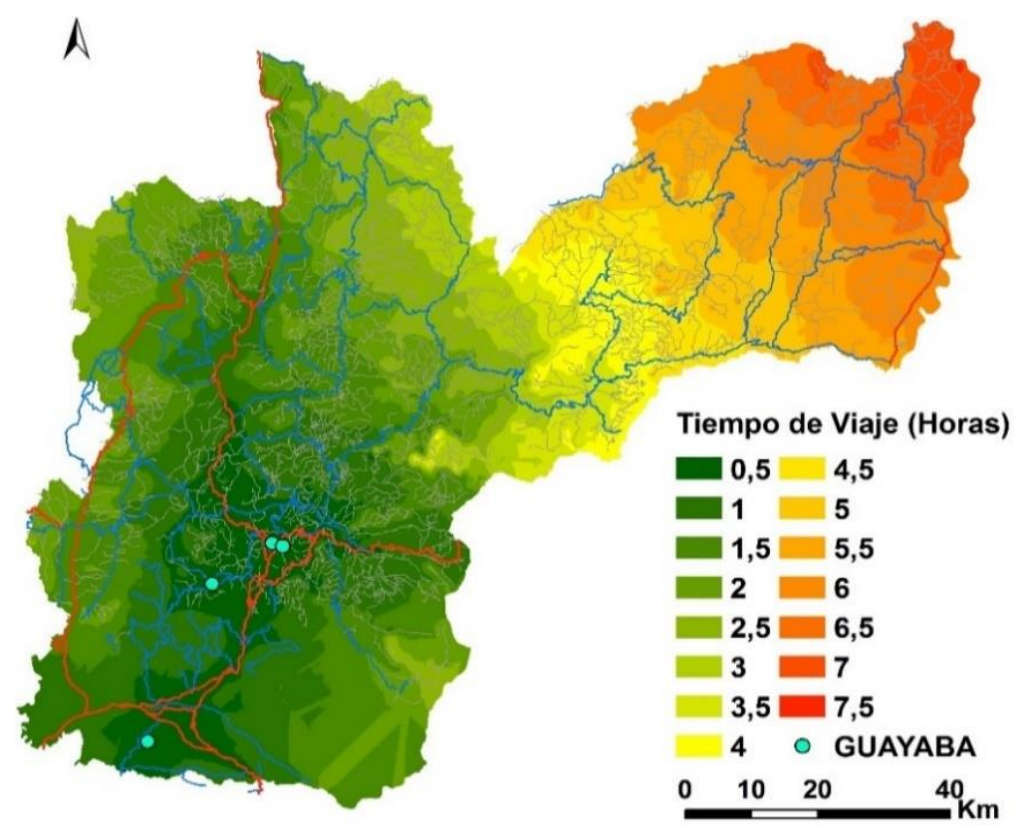

Fig. 6. Curvas isócronas hacia las fincas productoras de Guayaba

Por otra parte, el sector oriente (La Dorada) se encuentra cubierto por las curvas de entre 7 y 7,5 horas, indicando que deberá invertirse dicha cantidad de tiempo medio de viaje para alcanzar la finca productora de guayaba más próxima. Analizando la relación entre las características operativas de la red y las variables PIB, área y población (Ver Figura 7a), se encontró que las variables población y PIB refieren mayores porcentajes de cobertura para menores tiempos medios de viaje si se compara con los porcentajes de cobertura de la variables área, indicando que la ubicación de las fincas productoras de guayaba es cerca a ciudades como Manizales y Pereira, las cuales cuentan con los mayores valores de PIB y una alta densidad poblacional en relación con los demás municipios en consideración. Sin embargo, puede evidenciarse un cubrimiento superior al $60 \%$ de las tres variables invirtiendo hasta 2,5 horas de tiempo medio de viaje, lo cual indica que la red vial existente ofrece un alto nivel de cobertura en relación con la ubicación de las fincas productoras de guayaba. 
En la Figura $7 \mathrm{~b}$, desagregando los resultados de cobertura de la variable PIB, se encontró que para un $50 \%$ de cobertura de esta variable, son los municipios de mayor PIB (PIB6), los que refieren menores tiempos medios de viaje, aproximadamente 45 minutos, valor que para el caso de tomate de árbol se encontraba en 2,75 horas para el mismo tipo de PIB. Así mismo se tiene que para valores de tiempo medio de viaje superiores a 1 hora, la cobertura es mayor para todos los PIB menos para el PIB6, reforzando lo concluido con anterioridad en relación con la cercanía de estas fincas a importantes centros de comercialización. Los PIB 1 y 4 son los que refieren condiciones de accesibilidad más deficiente en relación con la ubicación de las fincas productoras de guayaba.
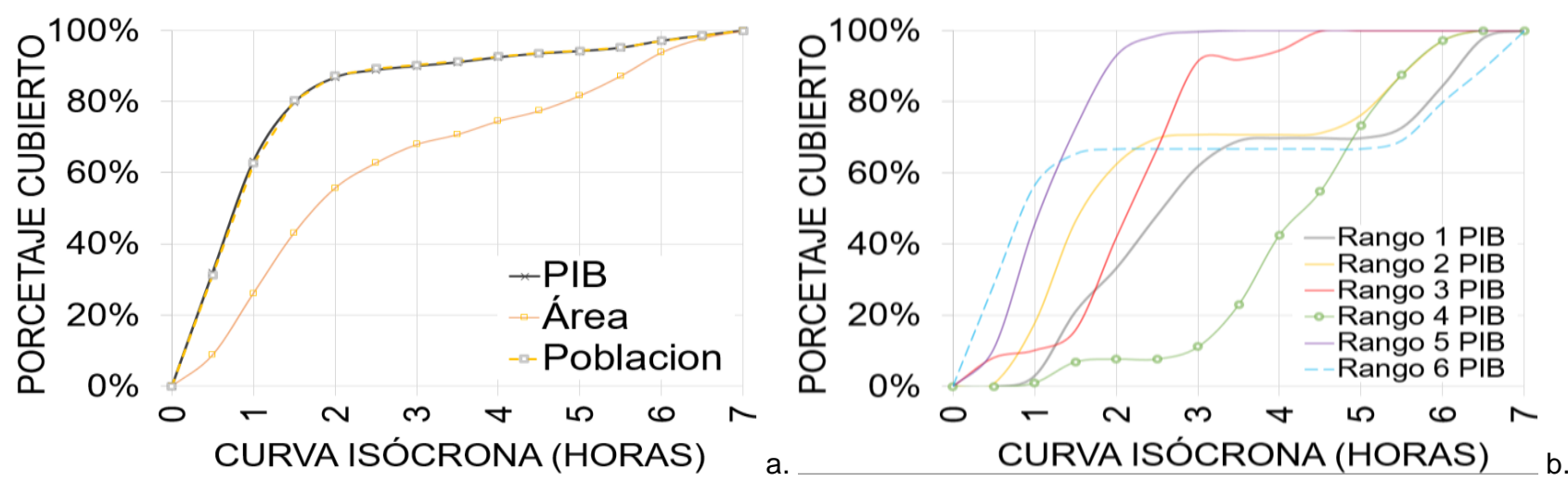

Fig. 7. a. Ojivas de cobertura de fincas productoras de Guayaba según PIB, área y población; b. Ojivas de cobertura según rangos de PIB.

\section{Fincas productoras de mora}

En la Figura 8 se observan las curvas isócronas calculadas para evaluar la accesibilidad geográfica hacia las fincas productoras de mora. Este es el producto predominante en las fincas productoras analizadas (14 fincas productoras), las cuales se localizan en el centro, norte y occidente del Departamento de Caldas, en su mayoría, circundando vías de categoría secundaria. Se pueden apreciar curvas que van desde las 0,5 horas hasta las 6,5 horas de tiempo medio de viaje. La zona del centro occidente caldense refiere las condiciones más favorables presentando tiempos de hasta 3,0 horas para los municipios más lejanos hacia el sur de la zona de estudio. Una vez más, desde el municipio de La Dorada se registran los mayores valores de tiempo medio de viaje.

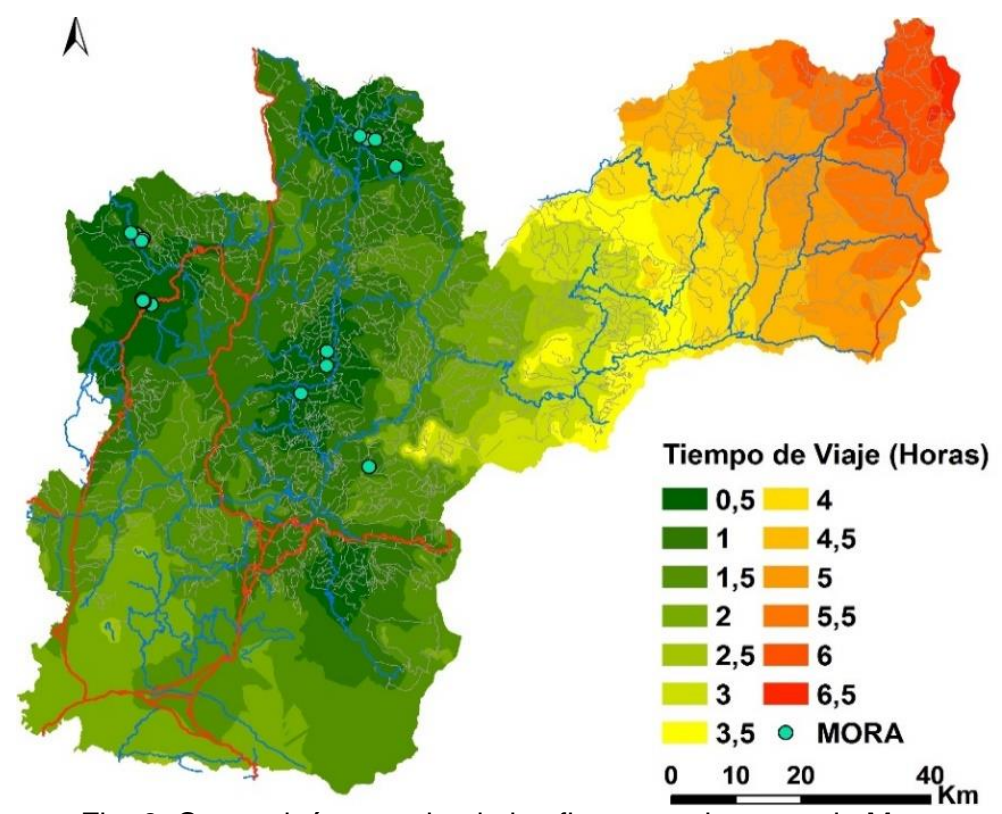

Fig. 8. Curvas isócronas hacia las fincas productoras de Mora

La relación entre las características operativas de la red y las variables PIB, área y población, se observa en la Figura 9a que para un valor de cobertura del $50 \%$ se registran tiempos medios de viaje inferiores a 1,5 horas. Sin embargo, para tiempos medios de viaje mayores, el porcentaje de cobertura de las variables PIB y Población sigue aumentando, pero no aumenta al mismo ritmo para la variable área, encontrando, por ejemplo que para 2 horas de tiempo medio de viaje, se registra una cobertura del $66 \%$ del área, mientras que 
para las otras dos variables la cobertura llega al $90 \%$; esto se debe a la cantidad de fincas productoras, a la dispersa ubicación de las mismas y a que las grandes zonas comerciales se encuentran más alejadas de éstas, si se compara, por ejemplo con las fincas productoras de Guayaba. Por otra parte, en la Figura 9b, al desagregar los resultados de cobertura de la variable PIB, se encontró que para un $50 \%$ de cobertura de esta variable, son los municipios de rango 3 y 5 , los que refieren menores tiempos medios de viaje (menor a 1 hora), situación semejante a la encontrada con las fincas productoras de tomate de árbol. Así mismo se tiene que para valores de tiempo medio de viaje superiores a 2 horas, la cobertura es mayor para los rangos de PIB 3 y 5, mientras que para los otros rangos dicho porcentaje de cobertura se estabiliza hasta un tiempo medio de viaje cercano a las 4 horas.
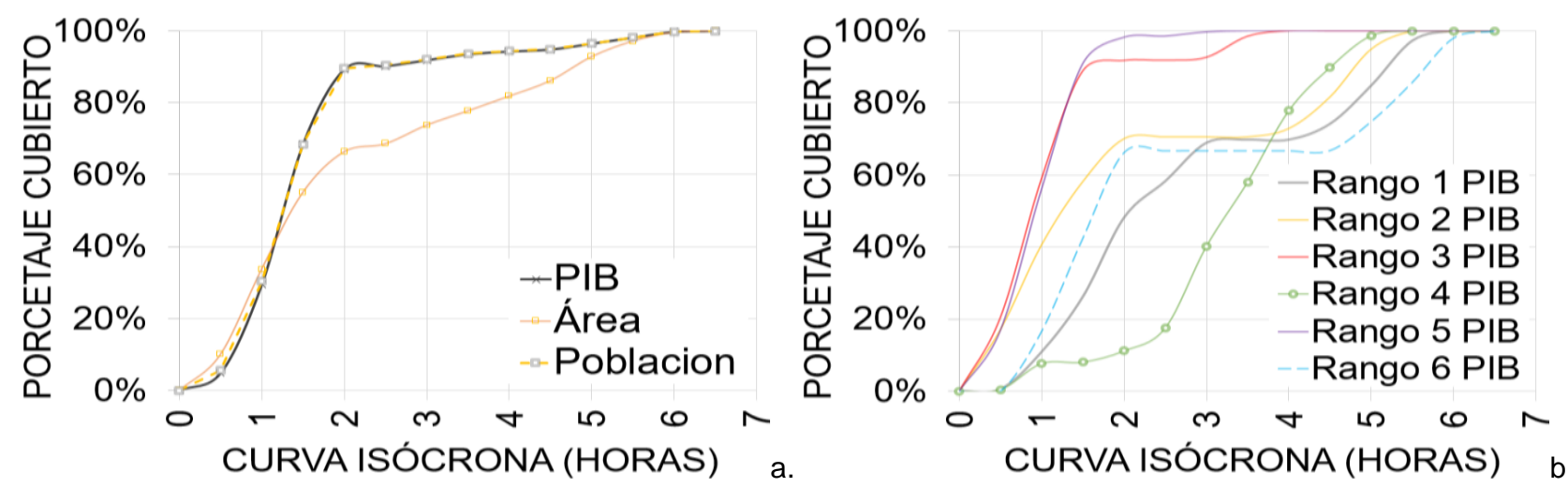

Fig. 9. a. Ojivas de cobertura de fincas productoras de Mora según PIB, área y población; b. Ojivas de cobertura según rangos de PIB.

\section{Fincas productoras de maracuyá}

Se tuvieron un total de cuatro fincas dedicadas a cosechar este producto, las cuales se ubican en zonas muy próximas a las vías principales, excepto una de ellas que se ubica circundante a una vía terciaria. En la Figura 10 se presentan las curvas isócronas obtenidas para esta fruta; se registran tiempos medios de viaje que van hasta las 7,5 horas. Se observa la forma como se extiende la curva correspondiente a 0,5 horas alrededor de un corredor de movilidad principal al occidente del área de estudio, en sentido norte - sur, lo cual seguramente aumenta la competitividad en relación con la comercialización de este producto. Los municipios ubicados en el departamento de Risaralda, así como los ubicados al norte del Departamento de Caldas presentan favorables condiciones de accesibilidad, registrando tiempos medios de viaje inferiores a las 3 horas; así mismo, nuevamente se evidencian desfavorables condiciones de accesibilidad geográfica hacia el oriente de la zona de estudio (municipios de Samaná, Norcasia, Victoria y La Dorada), en donde se presentan tiempos medios de viaje de entre 6,5 y 7,5 horas para alcanzar la finca productora de maracuyá más próxima.

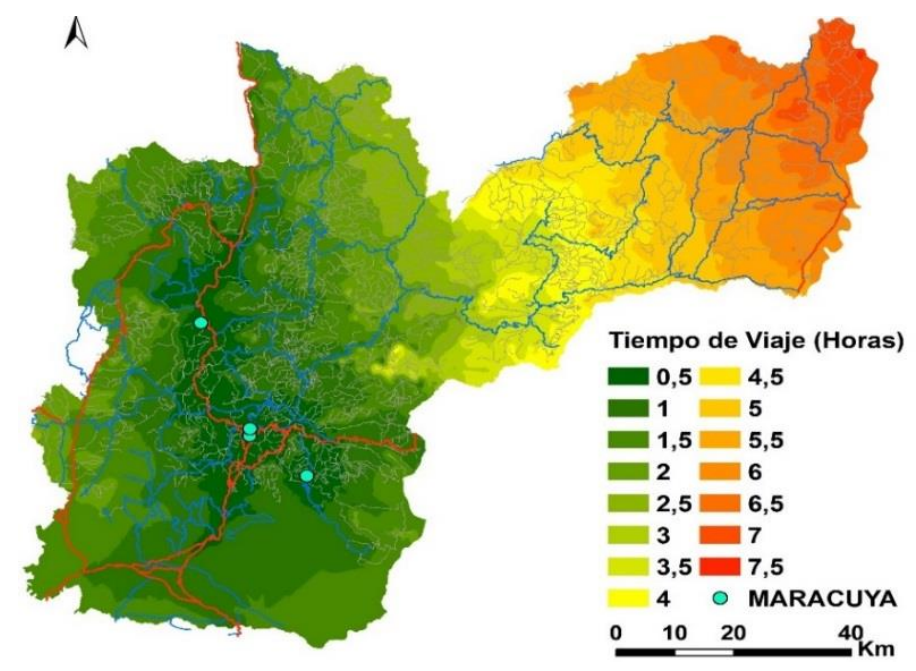

Fig. 10. Curvas isócronas hacia las fincas productoras de Maracuyá

Por otra parte, la concentración de este tipo de fincas alrededor del municipio de Manizales, explica la amplia cobertura que se presenta para las variables PIB y población, la cual es del $86 \%$ para tiempos medio de viaje inferiores a 1,5 horas mientras que empleando el mismo tiempo de viaje se abarca el $52 \%$ del área (Ver Figura $11 a)$, esto se debe a la proximidad existente entre las fincas productoras y las vías principales. En la Figura 
$11 \mathrm{~b}$, discretizando los resultados de cobertura de la variable PIB, se encontró que para un $50 \%$ de cobertura de esta variable, son los municipios de mayor PIB (PIB5 y PIB6), los que registran menores tiempos medios de viaje (entre 0,75 y 1,25 horas), situación semejante a la encontrada con las fincas productoras de guayaba. Por otra parte, se encontró que para valores de tiempo medio de viaje superiores a 1,5 horas, la cobertura es mayor sólo para los rangos de PIB 5 y 3 , mientras que para los otros rangos dicho porcentaje de cobertura se estabiliza hasta un tiempo medio de viaje próximo a las 4,5 horas.
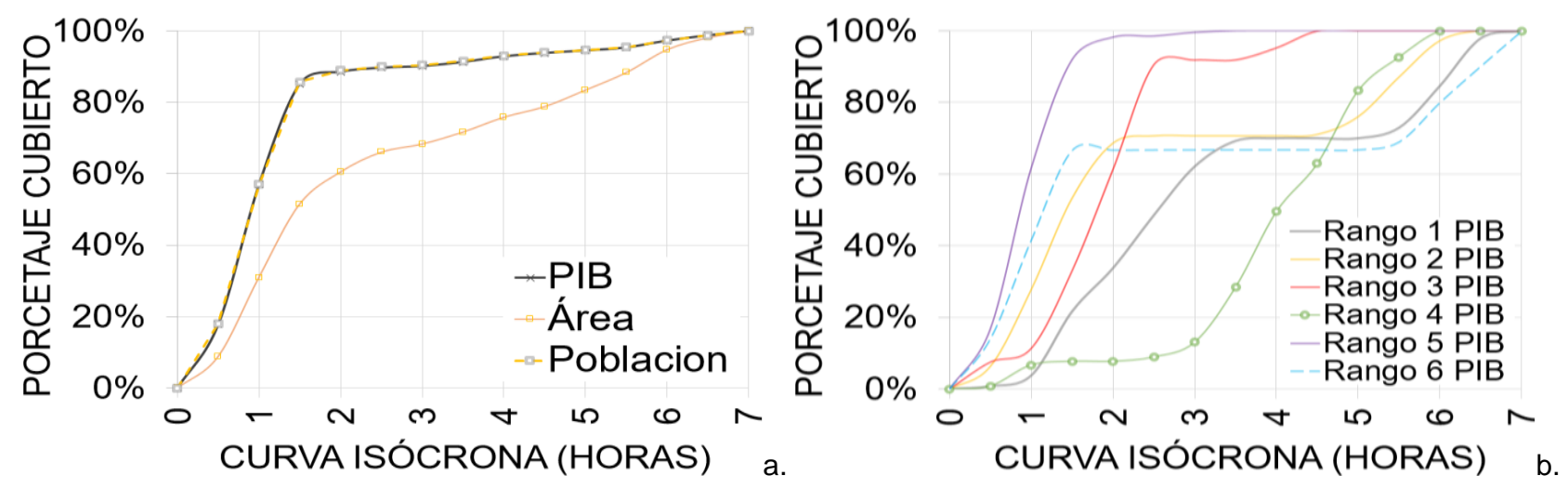

Fig. 11. a. Ojivas de cobertura de fincas productoras de Maracuyá según PIB, área y población; b. Ojivas de cobertura según rangos de PIB.

\section{Fincas productoras de lulo}

En la Figura 12 se presentan las curvas isócronas obtenidas para las siete fincas productoras de lulo que hacen parte del estudio; se identifican tiempos medios de viaje de hasta 7 horas para alcanzar alguna de ellas. De las fincas productoras, el $50 \%$ de éstas se ubican en la ciudad de Manizales, muy próximas a corredores viales de categoría primaria, lo cual se traduce en tiempos de viaje inferiores a 3 horas para toda la zona centro occidental del área de estudio. Al igual que con las demás fincas analizadas, se encuentra nuevamente que los municipios que se encuentran al otro lado de la cordillera central refieren las condiciones más desfavorables de accesibilidad, registrando tiempos de hasta 7 horas para llegar a la finca más cercana.

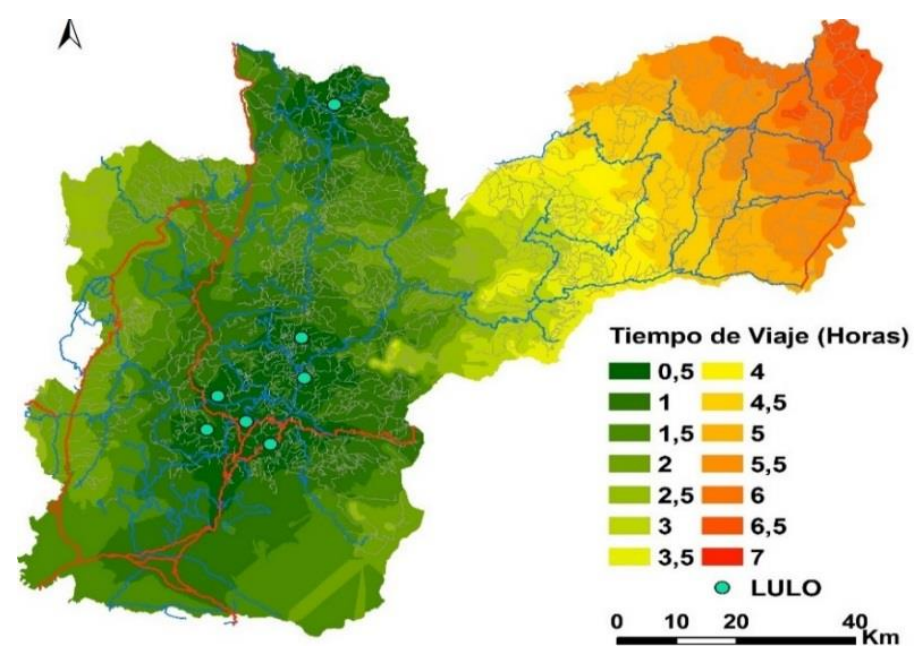

Fig. 12. Curvas isócronas hacia las fincas productoras de Lulo

Por otra parte, en la Figura 13a se observa la relación entre los tiempos medios de viaje y los porcentajes de cobertura de las variables área, población y PIB; se encontró que existe una cobertura del orden del $80 \%$ de la población y el PIB que cuentan con la posibilidad de acceder a una finca productora de lulo empleando un tiempo inferior a 1,5 horas, mientras que para el mismo tiempo de viaje se alcanza a cubrir tan sólo el $51 \%$ de la variable área; lo anterior, se debe a la alta concentración de fincas productoras de lulo en el municipio de Manizales, el cual posee un alto PIB y una importante densidad poblacional. En la Figura 13b, disgregando los resultados de cobertura de la variable PIB, se encontró que para un 50\% de cobertura de esta variable, son los municipios de mayor PIB (PIB5 y PIB6), los que registran menores tiempos medios de viaje (entre 0,7 y 1 horas), situación semejante a la encontrada con las fincas productoras de maracuyá y guayaba. Así mismo, se encontró que para valores de tiempo medio de viaje superiores a 2,5 horas, la cobertura es mayor sólo para los rangos de PIB 5 y 3 , mientras que para los otros rangos dicho porcentaje de cobertura se estabiliza hasta un tiempo medio de viaje próximo a las 5 horas. 


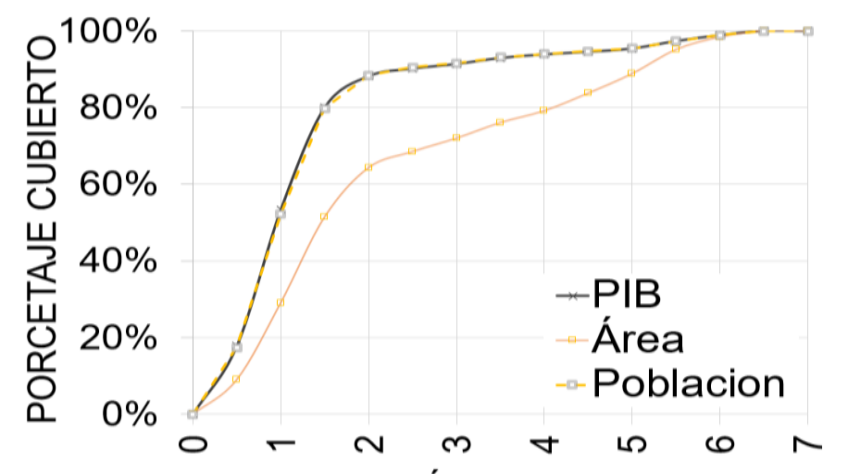

CURVA ISÓCRONA (HORAS)

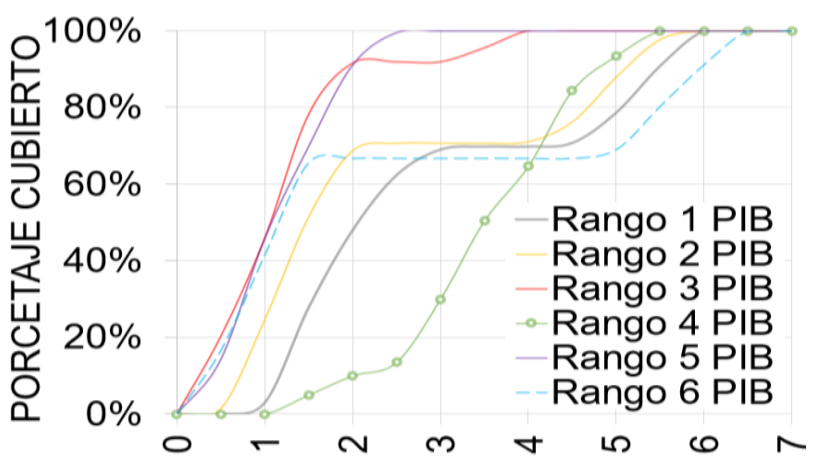

CURVA ISÓCRONA (HORAS) b.

Fig. 13. a. Ojivas de cobertura de fincas productoras de Lulo según PIB, área y población; b. Ojivas de cobertura según rangos de PIB.

\section{Análisis comparativo}

Analizando los resultados de cobertura obtenidos para la variable población y comparando según el tipo de fruta (Ver Figura 14), se tendría que para un $25 \%$ y un $50 \%$ de cobertura es la guayaba la fruta que se cubre con menor tiempo medio de viaje, con 0,40 y 0,79 horas, respectivamente, mientras que para un $75 \%$ de cobertura la fruta que se alcanza con menores tiempos medios de viajes es el Maracuyá con 1,28 horas de tiempo medio de viaje; así mismo, para el $25 \%, 50 \%$ y $75 \%$ de cobertura de la variable población, es el Tomate de Árbol la fruta que registra mayores tiempos medios de viaje con 2,05, 2,40 y 2,80 horas.

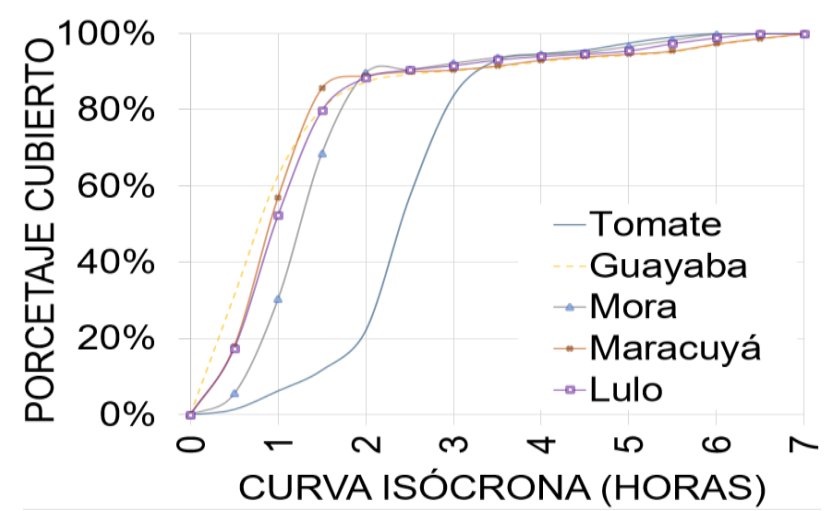

Fig. 14. Análisis comparativo respecto a la variable población

Por otra parte, para un $25 \%$ de cobertura de la variable PIB desagregada según el rango del mismo, se tiene que el menor tiempo medio de viaje registrado para alcanzar una finca productora se presenta a los 24 minutos (PIB 6) y el mayor tiempo medio de viaje para este porcentaje de cobertura se presenta a las 3,6 horas (PIB 4), en ambos casos para la Guayaba. Para un $50 \%$ de cobertura de la variable PIB desagregada, la fruta que registra el menor y el mayor tiempo medio de viaje también es la Guayaba con 0,9 horas (PIB 6) y 4,3 horas (PIB 4), respectivamente. Finalmente, para un $75 \%$ de cobertura, el menor tiempo medio de viaje es 1,2 horas (PIB 3) para la fruta mora y el mayor tiempo medio de viaje es 5,8 horas (PIB 6) para la fruta maracuyá.

Analizando la categoría de las vías cercanas a las frutas andinas estudiadas, se observa que son la Guayaba y el Maracuyá aquellas que tiene más cercanía a vías de categoría primaria, lo cual, en el caso de la Guayaba se relaciona directamente con los menores de tiempo medio de viaje registrados para diferentes valores de cobertura de las variables población y PIB. Es por ello que el desarrollo de la infraestructura del transporte se relaciona directamente con las políticas de planificación e impulso agroindustrial, en las cuales, el apoyo a este tipo de producción se encuentra claramente definido, mediante el incentivo dela producción de frutas libres de trazas de pesticidas, las cuales son nocivas para la salud, la implementación de buenas prácticas agrícolas tanto para la comercialización de los productos en fresco como para su conexión con procesos de transformación de la fruta como tal.

\section{CONCLUSIONES}

Se concluye que para las frutas estudiadas, la cobertura de la población entre el $25 \%$ y el $75 \%$ se alcanza invirtiendo entre 24 minutos (Guayaba) y 2,80 horas (Tomate de Árbol), los cuales son valores de tiempo medio de viaje que varían según la variable de análisis, por ejemplo, para el PIB 6 se reportan los menores tiempos medios de viaje, así como los mayores tiempos medios de viaje con 5,8 horas encontrados para el 
75\% de cobertura en la fruta maracuyá. La infraestructura del transporte actualmente disponible proporciona tiempos de viaje en algunos casos bajos y moderados hacia todas las fincas productoras, en consecuencia, puede inferirse que el proceso de traslado de los frutales andinos analizados no es lo suficientemente prolongado como para afectar su calidad.

Actualmente, la localización idónea de nuevas plantaciones frutales es en la parte occidental del Departamento de Caldas y en los municipios de Pereira, Santa Rosa de Cabal y Dosquebradas, ya que en estas zonas se encuentran los mejores indicadores de cobertura de las tres variables analizadas debido a la disponibilidad inmediata de vías de categoría primaria y secundaria. No obstante, si se desean fortalecer aún más las ventajas que actualmente representa la red vial para el transporte de productos frutales debe mejorarse la red vial terciaria, especialmente aquellos segmentos viales empleados como ruta de acceso y salida de las fincas productoras.

No se considera viable desde el punto de vista de movilidad, la implementación de cultivos en la zona oriente del departamento de Caldas, ya que la ausencia de corredores viales primarios incrementaría los costos de producción y afectaría la calidad de los frutales comerciales andinos estudiados. El oriente del departamento de Caldas requiere la construcción de infraestructura vial primaria que brinde conexiones expeditas con la región occidental de este departamento con el objetivo de impulsar de manera conjunta el desarrollo económico regional. Para futuras investigaciones, puede analizarse el beneficio que significará la implementación de nuevos proyectos viales, tales como la doble calzada que se proyecta entre Manizales y Medellín. Dado lo anterior, se concluye que este tipo de investigaciones en las que se aplican nuevas metodologías de priorización de intervenciones infraestructurales o de producción, apoyan de forma directa las diferentes políticas de planificación regional, que actualmente ejecuta la Gobernación de Caldas en relación con la agroindustria del departamento en tres proyectos puntuales: i) alianzas productivas, buscando la vinculación de pequeños productores rurales con mercados de transformación a través del esquema de agronegocio; ii) origen caldas, proyecto que busca impulsar y dinamizar la comercialización de productos del departamento; iii) seguridad alimentaria, proyecto mediante el cual se busca la apropiación, y soberanía alimentaria de la agro diversidad en el departamento. Dichos proyectos, en relación con la investigación, se abordan con el fin de atender políticas de localización de emprendimientos agrarios, entendiendo, por ejemplo que el tomate de árbol necesita más soporte infraestructural que las otras cuatro futas al ver la necesidad de una conexión más expedita con el área de análisis; así como atenderán políticas de industrialización de producción agraria y políticas relacionadas con los proceso de conservación de los productos.

\section{AGRADECIMIENTOS}

Los autores agradecen el apoyo del Banco Interamericano de Desarrollo (BID), a través del Fondo Regional de Tecnología Agropecuaria (FONTAGRO) y de la Universidad Nacional de Colombia sede Manizales. Se agradece especialmente al Ing. Diego García Flórez por el soporte prestado durante toda la investigación.

\section{REFERENCIAS}

Batty, M., Accessibility: in search of a unified theory, doi:10.1068/b3602ed, Environment and Planning B: Planning and Design, 36(2), pp.191-194, (2009).

Escobar D., Cadena, C. y Salas, A., Cobertura Geoespacial de nodos de actividad primaria. Análisis de los aportes a la sostenibilidad urbana mediante un estudio de accesibilidad territorial, doi:10.14508/reia.2015.12.23.13-27; Revista EIA, 12(23), pp. 13-27 (2015a).

Escobar, D., García, F. y Tolosa, R., Diagnóstico de la Movilidad urbana de Manizales, 1ª Edición, Universidad Nacional de Colombia. Facultad de Ingeniería y Arquitectura, Manizales, Colombia (2012).

Fox, M., Transport Planning and the human activity approach. Journal of Transport Geography, 3, (2) pp. 105-116, (1995).

Geurs, K. y Van Wee. Accessibility evaluation of land-use and transport strategies: review and research directions, doi: 10.1016/j.jtrangeo.2003.10.005, Journal of Transport Geography, 12 (2), pp. 127-140, (2004).

Goovartes, P., Geostatistics in soil science: state-of-the-art and perspectives, Geoderma. (89). pp. 1-45, (1999).

Gutiérrez, J., Condenço, A. y Martín, J., Using accessibility indicators and GIS to asses spatial spillovers of transport infrastructure investment, dioi: 10.1016/j.jtrangeo.2008.12.003, Journal of Transport Geography,(18), pp. 141-152. (2010).

Gutiérrez, J., Location, economic potential and daily accessibility impact of the high speed line Madrid-Barcelona- French border, Journal of Transport Geography, 9, pp 229-242. (2001).

Hansen, W., How accessibility shapes land use, doi:10.1080/01944365908978307, Journal of the American Institute of Planners, 25 (2), 73-76 (1959).

Hengl, T., Houvelink, G. y Rossiter D., About regression-kriging: From equations to case studies, Computers \& Geosciences, 33, pp 1301-1315. (2007). 
Kotavaara, O., Antikainen, H. y Rusanen, J., Population change and accessibility by road and rail networks: GIS and statistical approach to Finland 1970-2007, doi:10.1016/j.jtrangeo.2010.10.013 Journal of Transport Geography, 19 (4), pp. 926-935, (2011).

López, E., Gutierrez, J. y Gómez, G., Measuring regional cohesion effects of large-scale transport infrastructure investment: an accessibility approach, doi: 10.1080/09654310701814629, European Planning Studies, 16 (2), pp. 277301, (2008).

Mackinnon, D., Pirie, G. y Gather, M., Transport and economic development. In R. Knowles, J. Shaw, \& I. Docherty, Editors, Transport Geographies: Mobilities, Flows and Spaces (10-28). Blackwell Publishers, Oxford, (2008).

Martínez, S., Escobar, D. y Tamayo, J., Evaluación Comparativa de dos Alternativas de Infraestructura Tipo Túnel a partir de un Análisis de Accesibilidad Urbana, doi:10.4067/S0718-07642017000400018. Inf. Tecnol. [online]. 28(4), pp.157-168 (2017).

Mitchell, R. y Rapkin, C., Urban Traffic: A Function of Land Use, Columbia University Press, New York, (1954).

Montoya, J., Escobar, D. y Zuluaga, J., Acceso peatonal y cobertura de las estaciones del sistema de bicicletas públicas de la ciudad de Manizales, Revista Espacios, ISNN 0798-1015, 38(29), pp. 8 (2017).

Morris, J., Dumble, P. y Wigan, M., Accessibility indicators in transport planning, http://projectwaalbrug.pbworks.com/f/Transp+Accessib+-+Morris,+Dumble+and+Wigan+(1979).pdf; Transportation Research, A, 13, pp. 91-109 (1978).

Parde-Igúzquita, E. y Atkinson, P. Modelling the semivariograms and cross-semivariograms required in downscaling cokriging by numerical convolution-deconvolution, Computers \& Geosciences, 33, pp 1273-1284. (2007).

Park, S., Measuring public library accessibility: a case study using GIS, doi:10.1016/j.lisr.2011.07.007, Library \& Information Science Research, 34 (1), pp. 13-21 (2012).

Phatarapon V., Pongtep P., Suparda K y Nuchananporn P., A comparison of Spatial Interpolation Methods for predicting concentrations of Particle Pollution (PM10), International Journal of Chemical, Environmental \& Biological Sciences (IJCEBS), 3(4), pp.302-306, (2015).

Rietveld, P. y Nijkamp P., Transport and regional development. In: J. Polak and A. Heertje, Editors, European Transport Economics, European Conference of Ministers of Transport (ECMT), Blackwell Publishers, Oxford. (1993).

Van Zeebroeck, M., Tijskens, E., Dintwa E., Kafashan J., Loodts J., Baerdemaeker, J. y Ramon, H. The discret element method (DEM) to simulate fruit impact damage during transport and handling: Case study of vibration damage during apple bulk transport. Postharvest Biology and Technology, 41, pp. 92-100. (2006).

Van Zeebroeck, M., Van linden, V., Ramon, H., De Baerdemaeker, J., Nicolaï, B.M. y Tijskens, E. Impact damage of apples during transport and hanling. Postharvest Biology and Technology, 45, pp 157-167. (2007).

Vega, A., A multi-modal approach to sustainable accessibility in Galway, doi: 10.1080/20429843.2011.9727923, Regional Insights, 2(2), pp. 15-17, (2011).

Younes, C., Escobar, D. y Holguín, J., Equidad, Accesibilidad y Transporte. Aplicación explicativa mediante un Análisis de Accesibilidad al Sector Universitario de Manizales (Colombia), doi:10.4067/S0718-07642016000300010. Inf. Tecnol. [online]. 27(3), pp. 107-118 (2016).

Zuluaga, J. y Escobar, D., Geomarketing Analysis for Shopping Malls in Manizales (Colombia). Accessibility approach methodology, Revista Espacios, ISNN 0798-1015, 38(21), pp. 20 (2017). 